\title{
Tumores del tórax en niños
}

En el presente trabajo se analiza la experiencia del Centro Cardiovascular del Hospital Calvo Mackenna, en la patología tumoral torácica del niño; esta revisión comprende los casos que han sido atendidos en nuestro servicio en el periodo 1945-1975.

Comprende 35 niños afectados de esta patología con franco predominio de los tumotes y quistes del mediastino (Tabla $\mathrm{N}$ : 1).

\section{TUMORES DEL PULMON}

Su frecuencia comparada con otros tumores infantiles $o$ con los tumores pulmonares del adulto es insignificante. Los tumores malignos secundarios por metástasis del Tumor de Wilms, el neuroblastoma o el sarcoma osteogénico son más frecuentes que los primarios (7).

En nuestra serie observamos 8 niños con tumores pulmonares, la mayoría de los cuales son metastásicos, principalmente a partir del Tumor de Wilms (Tabla № 2).

La frecuencia por edad indica la máxima presentación entre los tres y seis años, siendo Ios varones los más afectados. Había un recién nacido, un niño de 3 años, un nī̄o de 4 años, 2 niños de 5 años, 2 niños de 6 años y un niño de 11 años.

\footnotetext{
- Becado. Serv. Cirugia Infantil y Ortopedia. Hosp. Roberto del Río.

**Centro Cardiovascular. Hosp. Calvo Mackenna. **Centro Oncología. Hosp. Calvo Mackenna.
}

Dres. Tomás Pruyas," Oscar Gómez,"* Claudio Arretz,** Helmuth Jaeger,"* y H. del Pozo.***

Con respecto al cuadro clínico, sintomatología respiratoria se observó en siete pacientes (tos, disnea, derrame pleural principalmente), acompañados de signos de compromiso del estado general en cinco de ellos (fiebre, decaimiento) con $S$. de Horner unilateral en un caso. Un niño cursó asintomático de sus metástasis pulmonares, siendo su hallazgo por control radiológico.

Entre los tumores secundarios, el término medio de tiempo en la producción de las metástasis, a partir del diagnóstico de la lesión primaria fue de nueve meses, oscilando entre los tres años y medio y la presencia simultánea del tumor abdominal con metástasis pulmonares bilaterales.

En dos niños se realizo tratamiento quirúrgico exclusivamente con extirpacion total de las lesiones, pólipo traqueal y hemangiopericitoma. En los otros seis niños, Tumor de Wilms y rabdomiosarcoma, se practico tratamiento combinado, cirugía, quimioterapia y radioterapia.

Cinco niños afectados de tumores malignos fallecieron con un promedio de sobrevida de seis meses, con respecto a los otros dos, uno con hemangiopericitoma maligno no volvió a control, estanđo viva una niñ̃a a los tres años y medio de ser operada de metástasis pulmonares de Tumor de Wilms.

\section{TUMORES Y QUISTES DEL MEDIASTINO}

En los niños la mayoria de los tumores intratorácicos están localizados en el mediasti- 
no o adyacentes a él. Ello es lo que sucedio en nuestra serie, siendo los tumores neurogénicos, los linfomas y los teratomas los más frecuentes en ese orden. Corresponden 13 a tumores malignos y 10 a tumores benignos (Tabla N: 3).

\section{CLASIFICACION}

Desde el punto de vista diagnóstico, el reconocimiento radiológico de los tumores mediastínicos es de la mayor importancia. En particular, la determinación exacta de la situación de un tumor en el espacio mediastinico permite extraer amplias conciusiones acerca de sut constitución anatomopatológica, de real valor en la evaluación preoperatoria a tealizar (2).

El mediastino se divide en tres áreas basadas en estructuras anatómicas discernibles tal como se perciben en una radiografía lateral del tórax:

1.- Anterior, entre la cara posterior del esternón, la entrada torácica y la cara anterior del corazón; allí se localizan los teratomas y los tumores del timo.

2,- Medio, entre la cara anterior del corazón y el borde anterior de los cuerpos vertebrales. Se localizan principalmente los infomas y las duplicaciones quísticas.

3.- Posterior, que corresponde a los canales paravertebrales, donde se localizan los tumores neurogénicos.

\section{TUMORES DEL MEDIASTINO POSTERIOR}

Los tumores neurogénicos fueron los más frecuentes, ubicándose los siete en el canal paravertebral (Tabla No 4).

La máxima frecuencia se presentó en niños menores de cuatro años, predominando francamente en las niñas.

La sintomatología principal correspondió a 1a respiratoria en todos los casos (neumonia, dolor torácico), acompañada de compromiso importante del estado general (pérdida del peso, decaimiento, fiebre, anorexia). Tres ni- nos tuvieron síntomas neurológicos, dos paresia de las extremidades inferiores por metás. tasis al espacio epidural y uno $\mathrm{S}$. de Horner por compromiso del simpático cervical. El tratamiento realizado consistió en la extirpación quirúrgica total en dos niños afectados de ganglioneuroma; en cuatro nirios con ganglioneuroblastoma y netroblastona, extirpación quirúrgica parcial, quimiotcrapia y radioterapia. En el restante caso no se reatizó tratamiento alguno por evolución sobreaguda del proceso. De los cuatro pacientes con tumores malignos en los que se realiz 6 tratamiento, tres se encuentran vivos en controles posteriores al año de operados.

\section{TUMORES DEL MEDIASTINO MEDIO}

Los linfomas fueron los tumores más frecuentes, ubicándose los seis en esta localización (Tabla No 5).

Todos los niños afectados de linfomas eran mayores de cinco años habiendo paridad con respecto al sexo (dos niños de 1 año, 1 niño de 9 años y 1 niño de 10 años).

En los seis pacientes había compromiso del estado general, en cinco sintomas respiratorios (tos, disnea, dolor torácico), en cuatro alteraciones del SRE (adenopatías perifericas, hepatomegalia, esplenomegalia). Además dos presentaron osteoartropatía hipertró. fica y otros dos sindrome de compresión de la vena cava superior.

En tres niños se practicó tratamiento quirúrgico con extirpación parcial de las lesiones en dos y total en uno; en los otros tres se limitó a la biopsia por la irresecabilidad de los tumores.

Quimioterapia y radioterapia fueron la base del tratamiento.

Cuatro niños fallecieron con un promedio de sobrevida de cinco meses. Uno no volvió a control y otro fue operado recientemente, siendo su control breve.

Con respecto a los tres quistes broncogénicos, los mismos fueron hallazgos de controles de rutina, ya que cursaron asintomáti- 
cos. Los niños afectados eran mayores de seis años, predominando los vatones. E1 tratamiento realizado consistió en la extirpación total de los quistes.

\section{TUMORES DEL MEDIASTINO ANTERIOR}

Los tumores más frecuentes fueron los teratomas encontrándose los cuatro en esa localización (Tabla No 6).

La distribución por edad fue irregular, siendo la mayoría varones.

En todos los casos se presentaron síntomas respiratorios (disnea, dolor torácico, neumonia); un niño de diez años con transformación maligna presentó pubertad precoz.

El tratamiento consistió en la extirpación total en los cuatro casos, asociándose quimioterapia en el teratocarcinoma, que falleció a los cinco meses de operado.

\section{TUMORES DE LA PARED TORACICA}

Son muy raros en los niños, siendo muy escasas las comunicaciones al respecto. En lo que están de acuerdo algunos autores es que en los niños los tumores malignos son más frecuentes que los benignos y que es más probable que sea maligno un tumor de la pared torácica en un niño que en un adulto (8). En nuestra pequeña serie de cuatro casos, todos fueron benignos, uno de localización esternal y tres costales (Tabla № 7).

Dos niños fueron menores de un año, habiendo paridad con respecto al sexo.

Los síntomas más frecuentes fueron el dolor y el tumor o deformación torácica.

Uno de los niños, el afectado de teratoma estetnal, presentó cardiopatía congénita asociada (atresia tricuspídea).

Se realizó tratamiento quirúrgico con extirpación total de las lesiones en los cuatro pacientes.
Tabla N: 1

TUMORES DEL TORAX EN NINOS

Centro Cardiovascular. Hospital Calvo Mackenna $1945 \cdot 1975$

Tumores del pulmon

Tumores y quistes mediastínicos $23 \quad 66 \%$

Tumores de la pared costal $4 \quad 11 \%$

Total $\quad 35 \quad 100 \%$

Tabla No 2

TUMORES DEL PULMON

\begin{tabular}{lll}
\hline Primarios & & $25 \%$ \\
Rabdotniosarcoma embrionario & 1 & \\
Pólipo angiomatoso traqueal & 1 & \\
Secundarios & & $75 \%$ \\
Tumor de Wilms & 5 & \\
Hemangiopericitoma maligno & 1 & \\
Total & 8 & $100 \%$
\end{tabular}

Tabla N: 3

TUMORES Y QUISTES DEL MEDIASTINO

\begin{tabular}{lrr} 
Tumores neurogénicos & 7 & $31 \%$ \\
Linfomas & 6 & $26 \%$ \\
Tumores teratomatosos & 4 & $17 \%$ \\
Quistes originados en el & & \\
intestino anterior & 3 & $13 \%$ \\
Tumores diversos & 3 & $13 \%$ \\
Total & 23 & $100 \%$ \\
\hline
\end{tabular}

Tabla N: 4

TUMORES DEL CANAL PARAVERTEBRAL

$\begin{array}{llc}\text { Tumores neurogénicos } & & 78 \% \\ \text { Genglioneuroma } & 2 & \\ \text { Ganglioneuroblastoma } & 2 & \\ \text { Neuroblastoma } & 3 & \\ \text { Tumores diversos } & & 22 \% \\ \text { Sarcoma indiferenciado } & 1 & \\ \text { Fibrohemangioma } & 1 & \\ \text { Total } & 9 & 100 \%\end{array}$


Tabla $N: 5$

TUMORES DEL MEDIASTINO MEDIO

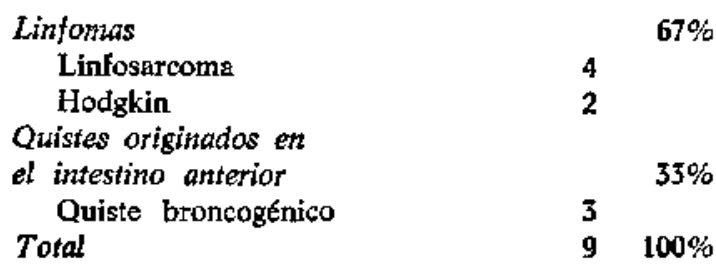

Tabla N

TUMORES DEL MEDIASTINO ANTERIOR

\begin{tabular}{lrr} 
Tumores teratomatosos & & \\
Teratome & 3 & \\
Teratocarcinoma & 1 & \\
Tumores del timo & & $20 \%$ \\
$\quad$ Hiperplasia del timo & 1 & \\
Total & 5 & $100 \%$ \\
\hline
\end{tabular}

Tabla N: 7

TUMORES DE LA PARED TORACICA

\begin{tabular}{lll} 
Tumores esternoles & & $25 \%$ \\
$\quad$ Teratoma & 1 & \\
Tumores costales & & $75 \%$ \\
Quiste aneurismático & 1 & \\
Fibroma & 1 & \\
Neurofibroma & 1 & \\
Total & 4 & $100 \%$ \\
\hline
\end{tabular}

\section{RESUMEN}

Se analizaron 35 niños afectados de tumores que se presentaron en el Centro Cardiovascular del Hospital Calvo Mackenna en el período 1945-1975.

Dentro de los tumores del pulmón, los primarios del mismo son extremadamente raros, predominando los metástasicos, sobre todo a partir del nefroblastoma.

Se destaca la mayor incidencia de los tumores y quistes del mediastino, siendo los neurogénicos los más frecuentes dentro de ellos.
Se demuestra la importancia de la $\mathrm{R} \times \mathrm{la}$ teral del torax en la localización y en la presunción diagnóstica preoperatoria consecuente.

El diagnóstico definitivo, sin embargo, es de exclusivo resorte de la histopatología, no debiéndose extender inútilmente el período de estudio y preparación pre-operatorios.

La evolución de los 15 niños con tumores benignos, en los cuales se realizó extirpación total fue excelente.

El tratamiento quirúrgico realizado en los 20 niños afectados de tumores malignos, consistió en la extirpación parcial en 9, total en 6 , y sólo biopsia en 5, por la irresecabilidad de las lesiones (esto más evidente en los linfomas).

Radioterapia se aplicó en 14 casos y quimioterapia se administro a 11 pacientes.

De los 20 niños con tumores malignos, 12 fallecieron, 2 no volvieron a control y actualmente se encuentran vivos 6 pacientes.

\section{SUMMARY}

We analize 35 cases of thoracic tumors seen at the Calvo Mackenna Hospital, Cardiovas. cular Center, since 1945 to 1975.

Lungs primary tumors are very unusual, being more frequent metastatic tumors, generally from a nephroblastoma.

It is outstanding, the high incidence of tumors and cysts of mediastine, being more frequent the neurologic ones.

It is very important a lateral chest $\mathrm{X}$ ray film in order to determine the localization, wich generally gives a reasonable diagnostic approach before the operation.

The final diagnostic is only based en histological studies. There must not be time lest with long studies and patients must be sent to surgery as soon as possible.

There were 15 bening tumors, that were complety resected. All patients are doing well up to date.

The surgical treatment in $\mathbf{2 0}$ malignant tumors, was partial resection in 9, total resection in 6 and only biopsy in 5 patients, with too large lesions (specially lynphomas). 
Radiumtherapy was used in 14 cases and chemiotherapy in 11 patients.

Regarding the 20 patients with malignant tumors, 12 are death, there is not control in 2, and 6 are alive.

\section{REFERENCIAS}

1. Filler, $R$. et al.: Favorable outlook for children with mediastinal neuroblastoma. J. Pediatr. Surg. 7: 136-143, 1972.

2. Pokorizy, W; Sharmann, $J:$ Mediastinal masses in infants and children. J. Thorac. Cardiovasc. Surg. 68: 869.875, 1974.

3. Haller, J. et al: Life-Threatening Respiratory Distress from Mediastinal Masses in Infants. Ann. Thorac. Surg. 19: 364-370, 1975.

4. Haller,. , et al.: Diagnosis and management of mediastinal masses in children. J. Thorac. Cardiovase. Surg. 58: 385, 1969.
5. Heimburger, 1. et al.: Primary mediastinal tut mors of childhood. J. Thorac Cardiovascular Surg. 50: 92, 1965.

6. Williams, I.: Tumores en el Niffo. Barcelona. Ed. Pediátrica, 1973.

7. Benson $C$, et. al.: Cirugía Infantil. Barcelona. Salvat Edit., 1967.

8. Grob, $M$. et al.: Patología Quirúrgica Infantil. Barcelona. Javier Morata, 1958.

9. Rubush, J. et al.: Mediastinal tumors: Review of 186 cases. J. Thorac. Cardiovasc. Surg. 65: 216-222, 1973.

10 Vogt, Moykopf and R. Daum.: Mediastinoscopy in infancy and childhood. Z. Kinderchir. 10: 25-29, 1971.

11 Piramoon, A.M. et al.: Mediastinal enterogenic cyst with spinal cord compression. I. Pediatr. Surg. 9: 543, 1974.

12. Thompson, D.P. and T.C. Moore: Acute thoracic distress in childhood due to spontaneours rupture of a large mediastinal teratoma $\mathrm{J}, \mathrm{Pe}$ diatr. Surg. 4: 416-423, 1969. 